Article

\title{
Understanding the Transit Market: A Persona-Based Approach for Preferences Quantification
}

\author{
Gamal Eldeeb * and Moataz Mohamed $(1)$ \\ Department of Civil Engineering, McMaster University, Hamilton, ON L8S4L8, Canada; \\ mmohame@mcmaster.ca \\ * Correspondence: eldeebg@mcmaster.ca
}

Received: 14 April 2020; Accepted: 3 May 2020; Published: 9 May 2020

\begin{abstract}
The study aims at utilizing a persona-based approach in understanding, and further quantifying, the preferences of the key transit market groups and estimating their willingness to pay (WTP) for service improvements. The study adopted an Error Component (EC) interaction choice model to investigate personas' preferences in a bus service desired quality choice experiment. Seven personas were developed based on four primary characteristics: travel behaviour, employment status, geographical distribution, and Perceived Behavioural Control (PBC). The study utilized a dataset of 5238 participants elicited from the Hamilton Street Railway Public Engagement Survey, Ontario, Canada. The results show that all personas, albeit significantly different in magnitude, are negatively affected by longer journey times, higher trip fares, longer service headways, while positively affected by reducing the number of transfers per trip and real-time information provision. The WTP estimates show that, in general, potential users are more likely to have higher WTP values compared to current users except for at-stop real-time information provision. Also, there is no consensus within current users nor potential users on the WTP estimates for service improvements. Finally, shared and unique preferences for service attributes among personas were identified to help transit agencies tailor their marketing/improvement plans based on the targeted segments.
\end{abstract}

Keywords: persona-based approach; error component model; service desired quality; willingness to pay; shared and unique preferences

\section{Introduction and Background}

Luring people out of their cars into public transit is vital for making cities liveable, sustainable, and equitable. Less car-dependent travel behaviour is essential in mitigating congestion and pollution problems in our cities. Transit agencies and decision-makers are striving to understand customers' needs, and hence increasing ridership. As such, there is a continued emphasis in the literature on investigating the desired quality from transit services, such as the work of [1-3]. However, understanding transit service desired quality for a wide spectrum of nontransit users is equally vital to increase transit market share and reduce car dependency.

In this regard, the transit market is often classified, among other classifications, into current and potential transit users [4-7], and/or captive and choice users [8-11]. Choice users have access to multiple modes of travel, while captive users have access to only one mode of travel. A spatial segmentation approach is also adopted to understand the preferences of different groups of transit users from a geographical perspective [12-14]. Additionally, other studies adopted a cluster analysis approach to extract homogenous customer groups with respect to preferences towards transit service quality $[12,15,16]$. All these prior classification approaches are often utilized to provide additional layers of information to better understand the preferences of different customer groups within the transit market. 
Other studies incorporated socioeconomic attributes and travel behaviour characteristics to understand the preferences of different user groups within the transit market, such as the work of [17], who utilized Multinomial Logit (MNL) Interaction Models, [18], who adopted an Ordered Probit Model (OPM), and [3], who employed Multiple Indicators Multiple Cause (MIMIC) Structure Equation Modelling (SEM) approaches. Their central goal, however, is rooted in understanding the heterogeneity in the preferences of transit customers.

In addition, discrete choice models, such as Mixed Logit (ML) Models and Latent Class Choice Models (LCM), are utilized in the literature to understand the broad span of preferences that exist in the transit market. Ventor [10] utilized an ML model to examine the stated preferences of captive and choice transit customers; the study proved the existence of preference heterogeneity within each user group. Also, the ML model was utilized in [19] to investigate observed and unobserved preference heterogeneity within transit users. ML and Latent Class (LC) choice models were utilized in [20] to investigate preference heterogeneity within current and potential transit users' groups, while Eldeeb and Mohamed [21] adopted ML and LC choice models to unveil preference heterogeneity within the whole transit market and classify the transit market into groups with homogenous preferences.

This mosaic of methodological techniques and user classification approaches share the same objective to better understanding the preferences towards transit service quality in a way that represents the entire population.

Nevertheless, recently, transit agencies opt to understand the transit market preferences based on a user-profile approach contrary to investigating the transit market based on independent socioeconomic attributes and/or travel behaviour characteristics. A user-profile approach allows transit agencies to target specific customer groups (i.e., key customer segments) and to consider specific real-life, easy-to-target customers. More specifically, transit agencies such as, among others, Metrolinx [22], TransLink [23], and EMT Madrid [24] are considering a persona-based approach to better understand their customers, as well as their travel behaviours and preferences. According to [25], a customer persona (detailed in Section 2) represents a group of targeted customers that share the same goals, needs, and behaviour.

For example, Metrolinx [22] developed six regional personas to better understand the travel behaviour and preferences of residents of the Greater Toronto and Hamilton Area (GTHA). Those personas are: (1) Time and Balance Seekers, (2) Traditional Suburban Travellers, (3) Frustrated Solution Seekers, (4) Connected Optimizing Urbanites, (5) Satisfied Mature Urbanites, and (6) Aspiring Young Travellers. A detailed description of each persona is available in the Metrolinx 2041 Regional Transportation Plan, Appendix 2D [22].

However, recognizing the scarcity of implementing the persona method in the transit quality literature, the authors argue on the pressing need of public transit agencies to better understand the preferences of the key market segments and advance the use of the persona-based approach beyond its current qualitative nature.

In this respect, the aim of this paper is twofold: (1) Understanding the preferences of the dominant transit market segments considering a persona-based (user-profile) approach, and (2) Advancing the use of the persona-based approach through quantifying personas' preferences and estimating their willingness to pay for service improvements. Accordingly, transit agencies should reconcile their marketing/improvement plans with a better understanding of their key customers' needs and based on quantified measures.

Towards that end, the study adopted an Error Component (EC) interaction choice model along with a persona-based approach in order to investigate shared preferences versus unique preferences associated with different transit market groups and quantify their willingness to pay (WTP) for service improvements. The study utilized a primary dataset of 5238 respondents elicited from an online survey that was part of Hamilton Street Railway (HSR) Public Engagement efforts in the city of Hamilton, Ontario, Canada. 
The remainder of this paper is arranged as follows: Section 2 provides a review of previous persona-based studies; Section 3 describes the modelling approach as well as the data collection process; Section 4 presents the results of the Error Component (EC) interaction model, which is followed by a discussion and concluding remarks in Sections 5 and 6, respectively.

\section{Persona-Based Approach}

A persona, first introduced by Cooper [26], is a user-centric design approach. The persona is defined as a fictitious character that portrays a targeted group of customers [26]. A customer persona represents a group of individuals who share common goals, needs, and behaviour [25]. From a practical approach, the customer persona method is used by designers, planners, and developers to identify, and later target, key customer segments. The persona method, as stated in [27], facilitates two major questions: first, who are we planning for? and second, who are we not planning for?

The method has been successfully implemented in understanding the actual goals of the targeted customers, prevention of self-referential design, and structuring research data in a more vivid form compared to raw data [27]. A step-by-step methodology of developing personas as a user-centred design method is provided by Nielson [28].

The persona-based approach has been adopted in many disciplines such as, among others, software development and webpage design to allow software/web developers to better understand prospective customers as well as their needs and requirements [29,30]. It was also implemented in automotive manufacturing to ensure that the design team has a common understanding of the customers' needs [31], and to examine different scenarios of vehicle design conception [32]. Further, in health sciences, the persona method was used to develop tailored health education messages to address patients' preferences [33] and to inform the design of a user-centred information resource regarding natural-products and conventional-drugs interaction [34]. In education, the persona approach is utilized as a pedagogical tool $[35,36]$.

In the transportation research literature, Lindgren et al. [37] used personas to identify the requirements of a dynamic graphical interface for Advanced Driver Assistance Systems (ADAS). Schäfer et al. [38] adopted the persona method in describing escape routes' users in subway stations to better picture their expectations and requirements. De Clerk et al. [39] employed a persona-based approach to assessing the balance between ownership and external costs associated with electric and conventional vehicle technologies. Kong et al. [40] employed personas to aid the design of human-robot interactions to build acceptance among various user types regarding the use of autonomous buses in mass transit.

However, to the best of the authors' knowledge, the use of the persona method in the transit quality literature remains rare.

Despite the aforementioned advantages, there are some limitations associated with the persona-based approach. The main limitation is the validity of the developed personas. It is argued that personas are hard to validate as they are developed based on the qualitative understanding of the important aspects of the final product/service [41]. Additionally, as argued in [42], the process of persona development might lead to base personas on stereotypes instead of genuine user types. However, the validity of the developed personas could be enhanced by using real data to inform the process of developing personas [43]. Another point to consider is that the prevalence rate (i.e., the proportion in the population) of a persona decreases with the addition of more attributes to describe each persona [44]. Nevertheless, the prevalence issue might be resolved through tuning down the number of attributes used in the persona development.

In this respect, the paper aims at utilizing the persona-based approach as a transit market taxonomy tool and advancing this approach by introducing quantified preferences and willingness-to-pay estimates for each persona. It is worth noting that the focus of this paper is not the development process of the personas; it is, rather, understanding and quantifying the preferences of the salient transit personas. 


\section{Methodology}

\subsection{Methods}

This study utilized an Error Component (EC) interaction model to investigate personas' preferences in a bus service desired quality choice experiment, and to estimate the influence of each attribute on the overall transit utility with respect to each persona.

The EC interaction model was used to independently investigate the preferences of the seven personas (explained in the next section), while accounting for the "panel effect" that emerged from the Stated Preference (SP) experiment. As one form of the Mixed Logit (ML) modelling family, the EC model was developed based on the works of $[45,46]$ and considered the Random Utility Maximization (RUM) theory [47,48]. For the EC model, the RUM adopts a rational decision-making approach, which assumes that individual $i$ picks the choice $j$ that maximizes their utility $U_{i j t}$, in the choice situation, $t$ :

$$
U_{i j t}=\beta X_{i j t}+\eta_{i j t} Y_{i j t}+\varepsilon_{i j t .},
$$

where $X_{i j t}$ is the observable component of the utility function, which is a vector of explanatory variables, and $\beta$ is a vector of estimated fixed parameters, while $\eta_{\mathrm{ijt}}$ is a vector of random elements with a distribution (assumed as normally distributed with zero mean), assigned by the modeller, and $Y_{i j t}$ is a vector of unknown attributes. $\varepsilon_{i j t}$ is the error term, which is assumed to be identically and independently distributed (IID).

The explanatory variables might include choice attributes as well as interaction variables reflecting the characteristics of each persona, which adopts the systematic taste variations specification suggested by [49,50]. The unconditional choice probability, as mentioned in [51], for individual $i$, selecting a choice $j$, based on the EC formulation, is expressed as follows:

$$
P_{i j}=\int \prod_{t=1}^{T_{q}}\left[\frac{e^{\beta X_{i j t}+\eta_{i j t} Y_{i j t}}}{\sum_{j=1}^{J} e^{\beta X_{i j t}+\eta_{i j t} Y_{i j t}}}\right] f(\eta) d(\eta) .
$$

The EC interaction model is estimated using a range of Modified Latin Hypercube Sampling (MLHS) draws (e.g., 50, 100, 500, 1000) through the Pandas Biogeme package [52]. According to Hess et al. [53], the MLHS outperforms other types of Quasi-random number sequences such as Halton draws. Since the choice experiment being dealt with is unlabelled, all alternative specific constants were excluded, and no respondents' specific attributes were introduced [54].

\subsection{Data and Survey Instrument}

The paper utilized a primary dataset from an online survey that was part of Hamilton Street Railway (HSR) Public Engagement efforts in the city of Hamilton, Ontario, Canada. HSR is the municipal public transit provider for the city of Hamilton and provides a service coverage area of 243 square kilometres through 35 regular bus routes [55]. The general purpose of the survey is to benchmark the service quality provided by HSR based on Hamiltonians' preferences and expectations. As mentioned in [56], the survey is designed for all Hamiltonians (i.e., both current and potential transit users) and structured in four independent sections: (1) Socioeconomic demographic characteristics and travel behaviour, (2) Stated Preference (SP) experiments, (3) Service quality aspects, and (4) Attitudinal and behavioural characteristics.

This paper utilized the unlabelled SP experiment, socioeconomic attributes, and travel behaviour characteristics components of the survey. The total number of respondents who answered the online survey by April 2019 was 5781 Hamiltonians. A total of 543 invalid, unengaged, and incomplete responses were removed based on a thorough validation process. Hence, a validated dataset of 5238 participants was utilized in the study. 
The design of the SP experiment employed, as advised by Bliemer and Rose [57], a three-stage sequential process (i.e., model specification, experimental design, and questionnaire) for the selection of the attributes and their associated levels. The final list of attributes and the associated levels are presented in Table 1.

Table 1. Unlabelled SP experiment attributes and their associated levels [56].

\begin{tabular}{cc}
\hline Service Attributes & Attribute Levels \\
\hline One-way trip cost & $\$ 3, \$ 4.50$, and $\$ 6$ \\
One-way trip travel time & 20,30 , and $40 \mathrm{~min}$ \\
Walking time to and from the bus stop & $0,5,10$, and $15 \mathrm{~min}$ \\
Service headway & $5,10,15$, and $30 \mathrm{~min}$ \\
Number of transfers & 0,1 , and 2 transfers \\
Real-time information & At-stop, onboard and none \\
\hline
\end{tabular}

The experimental design of the SP experiment adopted the efficient design approach, to improve the statistical efficiency and maximize the amount of information extracted from the SP experiment [58]. For the interested reader, a detailed description of the design process of the unlabelled SP experiment is introduced in [21,56]. Overall, the experimental design produced twelve scenarios grouped into three blocks. Each respondent faced four scenarios, and their choices were made from three unlabelled bus transit alternatives, as shown in Figure 1.

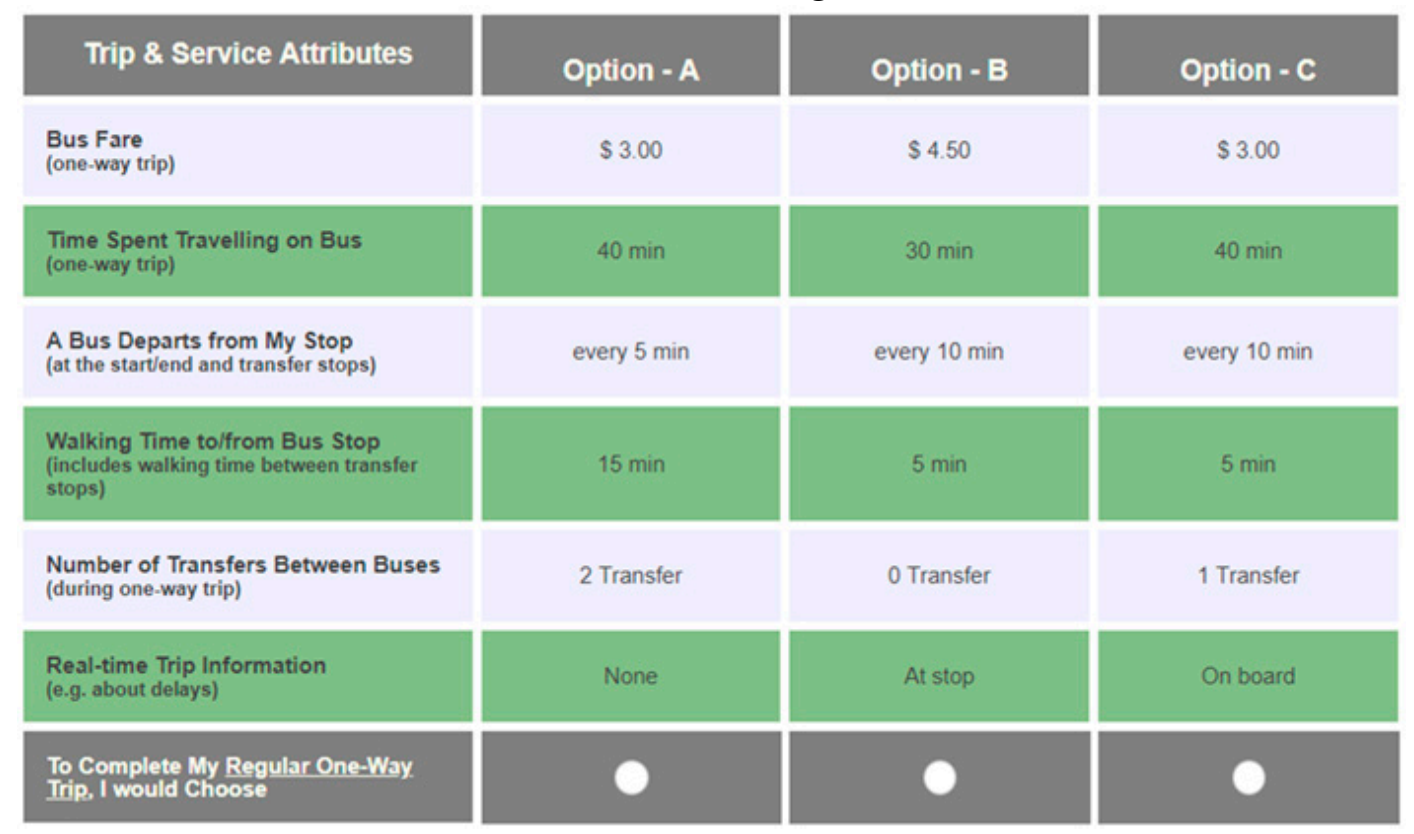

Figure 1. Example of a stated preference scenario in the survey [56].

In addition to the stated preferences, Perceived Behavioural Control (PBC) towards public transit was also utilized, which represents how easy/difficult respondents perceive transit use [59]. PBC was measured using three attitudinal statements: (1) Finding routes and schedules for my trip does not require too much effort, (2) It is easy to travel around the city using the HSR transit service, and (3) Transferring between routes is easy. Respondents were asked to assess their agreement on the accuracy of each statement on a 5-point Likert scale. These attitudinal statements have a Cronbach's alpha of 0.770 , which indicates internal consistency. 


\subsection{Adopted Personas}

Based on semistructured workshops with HSR personnel, the preliminary seven personas were identified. These personas are deemed to best describe the key groups of the targeted transit market within the city of Hamilton. The personas were developed, independently from the HSR public engagement survey, based on four main characteristics: travel behaviour, employment status, geographical distribution, and Perceived Behavioural Control (PBC).

The seven personas represent around $55.50 \%$ of our dataset; and the subsample includes 2907 respondents. Each persona portrays a typical group of Hamiltonians who are current or potential transit users as follows:

1. Persona 01 represents full-time employees who consider public transit as their primary mode of travel and are more likely to have a positive transit PBC and live in urban areas. This persona represents 912 respondents from the sample.

2. Persona 02 portrays students who rely on public transit as their primary mode of travel and are more likely to have a positive transit PBC and live in urban areas. This persona represents 526 respondents from the sample.

3. Persona 03 portrays full-time employees who live in urban areas, consider private vehicles as their primary mode of travel, and have more potential to have a neutral PBC. This persona represents 701 respondents from the sample.

4. Persona 04 depicts retirees who consider private vehicles as their primary mode of travel and are more likely to have a neutral transit $\mathrm{PBC}$ and live in urban areas. This persona represents 407 respondents from the sample.

5. Persona 05 represents students who consider private vehicles (driver or passenger) as their primary mode of travel and are more likely to have a neutral PBC and live in urban areas. This persona represents 142 respondents from the sample.

6. Persona 06 portrays full-time personnel who consider private vehicles as passengers their primary mode of travel and are more likely to have a neutral PBC and live in urban areas. This persona represents 83 respondents from the sample.

7. Persona 07 portrays full-time employees who live in the suburbs, identify private vehicles as their primary mode of travel, and are more likely to have a negative transit PBC. This persona represents 136 respondents from the sample.

Table 2 depicts the distribution of the personas' subsample, 2907 respondents, associated with different socioeconomic and demographic characteristics. The utilized sample represents more females $(57.93 \%)$ than males $(39.32 \%)$ and also includes $2.75 \%$ gender self-identity (e.g., prefer not to answer, non-binary, neutral, agender, transgender, etc.). Middle-aged respondents are the most represented in the sample $(50.33 \%)$, while old respondents are the least represented $(16.58 \%)$. Vehicle ownership ratio is relatively high, where about $83.21 \%$ of respondents have a vehicle in their household. Most respondents have a driver's licence $(78.57 \%)$ and live in urban areas $(83.56 \%)$.

Figure 2 summarizes the distribution of different socioeconomic and demographic characteristics for each persona. The highest percentages of males are in Persona 03 and Persona 04, while females are the highest in Personas 02, 05, and 06 categories. Among full-time personnel personas (i.e., Personas 01, 03, 06, and 07), the highest proportion of young respondents is in Persona 01, while the highest proportion of middle-aged respondents is in Persona 07. This corroborates that transit use is more prevalent among young full-time personnel compared to other age categories. The highest ratios of two or more vehicles in the household are in Persona 07 and Persona 05, while the highest ratio of one vehicle in the household is in Persona 06. Aside from Persona 07, the highest percentage of respondents who live in the suburbs are in Persona 04 (31.45\%) and Persona 05 (32.39\%). Regarding transit PBC, the highest percentage of respondents with a positive transit PBC is in Persona $01(60.16 \%)$, while the highest with a negative transit PBC is in Persona 07 (59.80\%). 
Table 2. Sample distribution.

\begin{tabular}{cccc}
\hline Category & Subcategory & Users (\%) & Population (\%) \\
\hline Total & Total & 2907 & $747,645(100 \%)$ \\
\hline \multirow{2}{*}{ Gender } & Male & $39.32 \%$ & $48.90 \%$ \\
& Female & $57.93 \%$ & $51.10 \%$ \\
& Self-identity & $2.75 \%$ & \\
\hline \multirow{2}{*}{ Age } & Less than 30 years old & $33.09 \%$ & $35.72 \%$ \\
& 30 to 59 years old & $50.33 \%$ & $40.64 \%$ \\
& Over 60 years old & $16.58 \%$ & $23.64 \%$ \\
\hline Vehicle ownership & Zero Vehicle & $16.79 \%$ & $13.00 \%$ \\
& One Vehicle & $41.04 \%$ & $87.00 \%$ \\
\hline Driver's licence & Two or more & $42.17 \%$ & - \\
& Holding & $78.57 \%$ & - \\
\hline Geographic distribution & Not holding & $21.43 \%$ & $36.69 \%$ \\
& Suburban areas & $16.44 \%$ & $63.31 \%$ \\
\hline
\end{tabular}

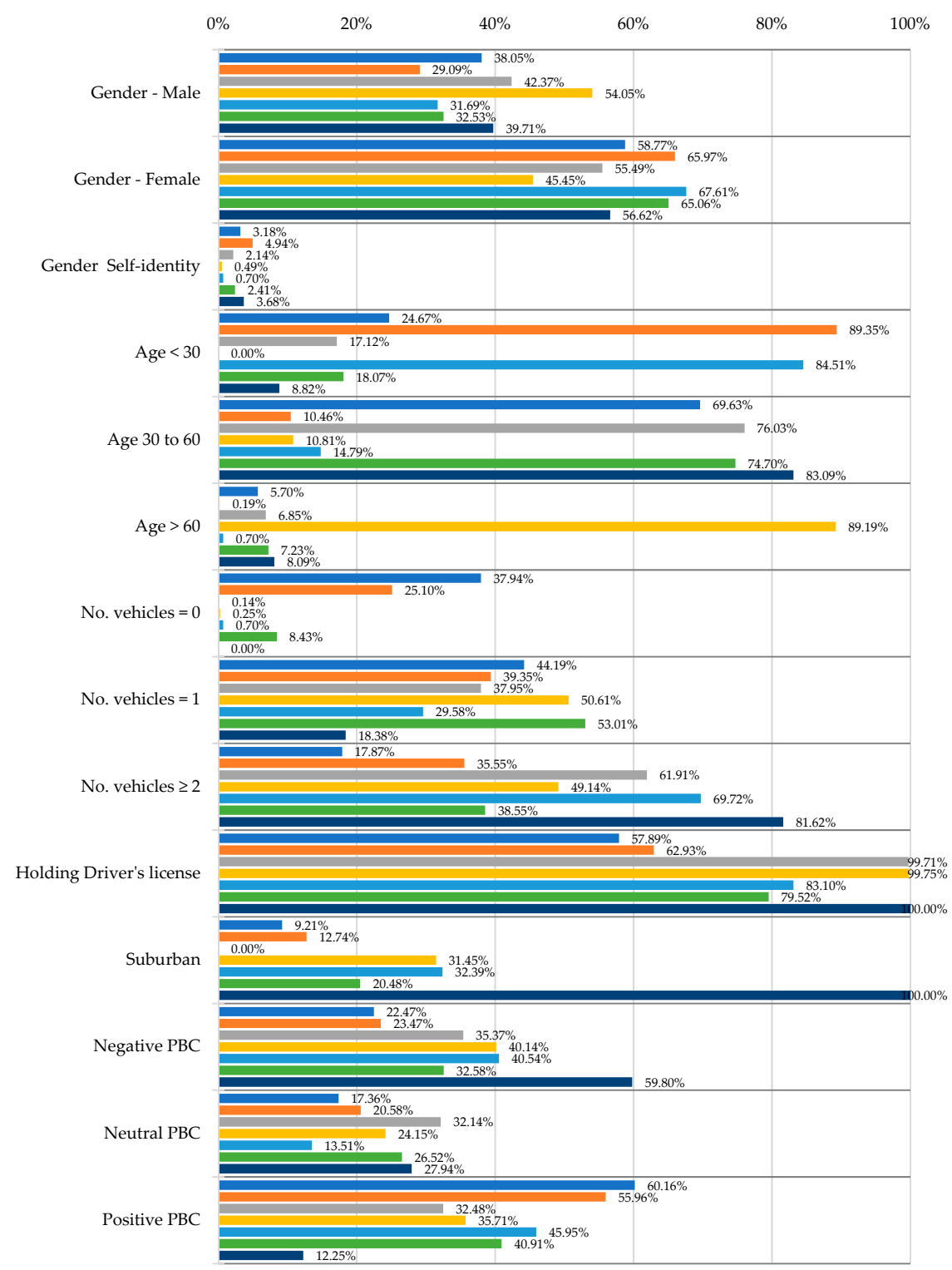

- Persona $01 \backsim$ Persona $02 \backsim$ Persona $03 \backsim$ Persona $04 \square$ Persona $05 \square$ Persona $06 \square$ Persona 07

Figure 2. The profile of the seven adopted personas. 


\section{Results}

\subsection{Persona-Based Preferences}

One inclusive EC interaction model was developed to estimate the influence of each attribute on the overall transit utility with respect to each persona and to explain personas' preferences and their statistically significant differences concerning service attributes. This, in turn, helps to identify the shared preferences versus the unique preferences associated with different transit market groups.

In our model, Persona 01 is considered the base category, and the results of the EC interaction model are shown in Table 3. The results include all personas' interactions with different service attributes in reference to Persona 01. The unique effects (coefficients) of service attributes with regard to each persona are presented in Table 4. The error component does not prove to be statistically significant; however, it is retained as a precautionary measure to account for the panel effect.

Table 3. Error component model estimates for personas' interactions.

\begin{tabular}{|c|c|c|c|c|c|c|c|}
\hline Variable & Persona 01 (Ref.) & $\begin{array}{l}\text { Persona } 02 \\
\text { Interaction }\end{array}$ & $\begin{array}{l}\text { Persona } 03 \\
\text { Interaction }\end{array}$ & $\begin{array}{l}\text { Persona } 04 \\
\text { Interaction }\end{array}$ & $\begin{array}{l}\text { Persona } 05 \\
\text { Interaction }\end{array}$ & $\begin{array}{l}\text { Persona } 06 \\
\text { Interaction }\end{array}$ & $\begin{array}{l}\text { Persona } 07 \\
\text { Interaction }\end{array}$ \\
\hline Journey time & $-0.041 * * *$ & 0.003 & $-0.015^{* *}$ & $0.017 * *$ & -0.005 & -0.014 & -0.011 \\
\hline Trip fare & $-0.541^{* * *}$ & 0.076 & $0.099 *$ & $0.184^{* * *}$ & $0.175^{*}$ & 0.142 & $0.235^{* *}$ \\
\hline Walking time & -0.007 & $-0.022 * *$ & $-0.035^{* * *}$ & $-0.022 * *$ & -0.017 & 0.004 & 0.002 \\
\hline Service headway & $-0.039 * * *$ & -0.003 & 0.005 & $0.027^{* * *}$ & $0.017 * *$ & 0.008 & 0.011 \\
\hline \multicolumn{8}{|c|}{ Number of transfers ( 2 transfers base category) } \\
\hline One transfer & $0.884^{* * *}$ & -0.005 & 0.010 & -0.115 & $-0.322 * *$ & -0.243 & $0.344^{* *}$ \\
\hline Zero transfer & $1.160^{* * *}$ & 0.032 & $0.384^{* * *}$ & -0.092 & -0.061 & 0.100 & $0.782 * * *$ \\
\hline \multicolumn{8}{|c|}{ Real-time information (No info. base category) } \\
\hline Real-time info. onboard & $0.388^{* * *}$ & 0.116 & -0.067 & -0.128 & 0.116 & -0.058 & 0.080 \\
\hline Real-time info. at-stop & $0.343^{* * *}$ & 0.026 & -0.124 & $-0.265^{* * *}$ & 0.143 & 0.040 & 0.046 \\
\hline Error component & & & & 0.016 & & & \\
\hline Log-likelihood & \multicolumn{7}{|c|}{$-11,580.86$} \\
\hline Log-likelihood ratio test & \multicolumn{7}{|c|}{2750.716} \\
\hline Rho-square & \multicolumn{7}{|c|}{0.106} \\
\hline
\end{tabular}

Note: ${ }^{* * *},{ }^{* *}$, and ${ }^{*}$ indicate significance at $1 \%, 5 \%$, and $10 \%$ levels, respectively, and Table A1-Appendix A presents the detailed results for the model estimates.

Table 4. The unique effects (coefficients) of service attributes with respect to each persona.

\begin{tabular}{cccccccc}
\hline Variables & Persona 01 & Persona 02 & Persona 03 & Persona 04 & Persona 05 & Persona 06 & Persona 07 \\
\hline Journey time & -0.041 & -0.039 & -0.057 & -0.025 & -0.046 & -0.055 & -0.052 \\
Trip fare & -0.541 & -0.466 & -0.442 & -0.357 & -0.367 & -0.400 & -0.306 \\
Walking time & -0.007 & -0.029 & -0.041 & -0.029 & -0.024 & -0.003 & -0.005 \\
Service headway & -0.039 & -0.042 & -0.034 & -0.011 & -0.021 & -0.030 & -0.028 \\
One transfer & 0.884 & 0.879 & 0.894 & 0.769 & 0.562 & 0.641 & 1.230 \\
Zero transfer & 1.160 & 1.190 & 1.540 & 1.060 & 1.100 & 1.260 & 1.940 \\
Real time info. onboard & 0.388 & 0.503 & 0.321 & 0.259 & 0.504 & 0.329 & 0.467 \\
Real time info. at stop & 0.343 & 0.369 & 0.219 & 0.078 & 0.486 & 0.382 & 0.388 \\
\hline
\end{tabular}

In the case of journey time, for all personas, transit utility is negatively affected by longer journey

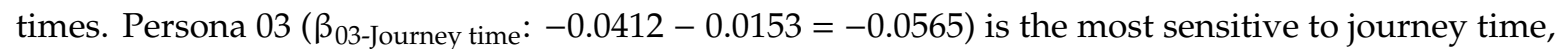

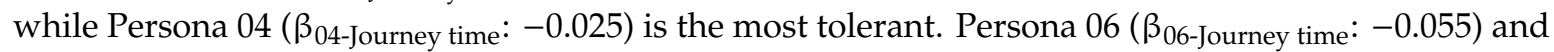

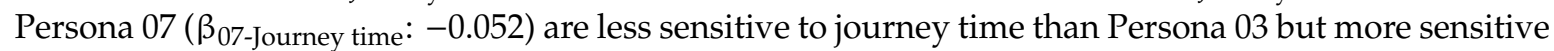

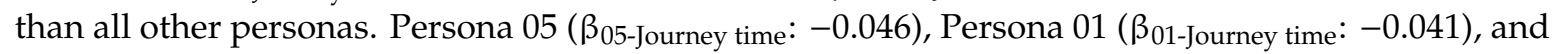

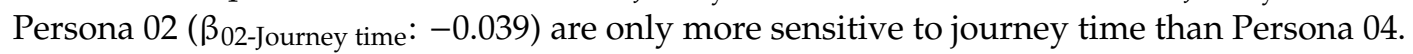

With respect to trip fare, the transit utility of all personas is negatively influenced by increasing

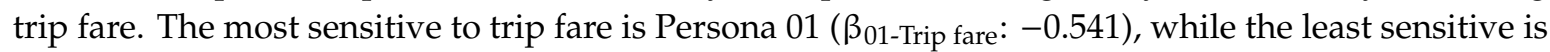

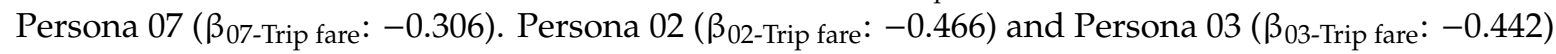
are more sensitive to trip fare than all other personas except Persona 01. Persona 06 ( $\beta_{06-T r i p ~ f a r e}$ :

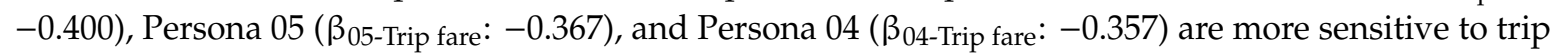
fare than Persona 07 and less sensitive than all other personas. 
Walking time to/from bus stop does not prove to significantly impact transit service utility for Personas 01, 05, 06, and 07, which implies that these personas are lenient regarding walking time to/from bus stops. Nonetheless, the most sensitive persona to walking time is Persona 03 ( $\beta_{03}$-Walking time: -0.041), while Persona 02 and Persona $04\left(\beta_{02}\right.$ \& \& 04-Walking time: -0.029$)$ are the second-highest most sensitive to walking time to/from the bus stop.

In regard to service headway (time between consecutive buses), the transit utility of all seven personas is negatively affected by less frequent transit services (higher headway). Persona 02

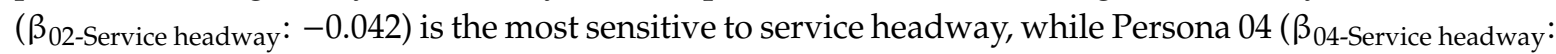

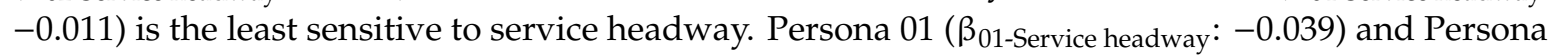

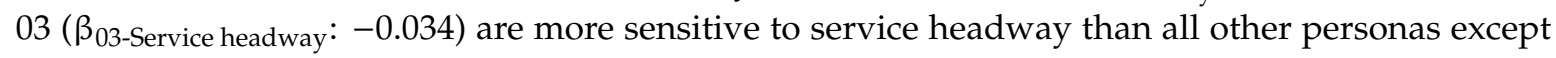

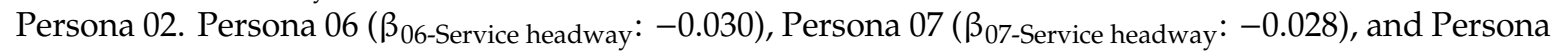

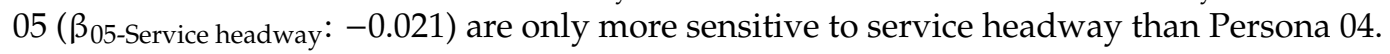

With reference to the number of transfers, all the seven personas are positively affected by reducing

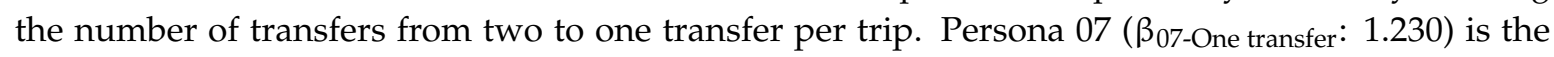
most influenced by reducing the number of transfers from two to one per trip, while Persona 05

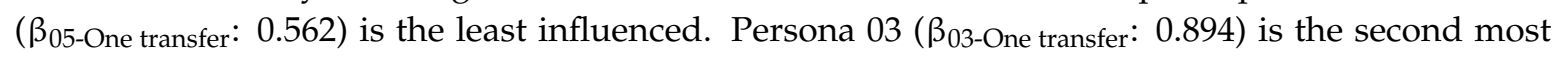

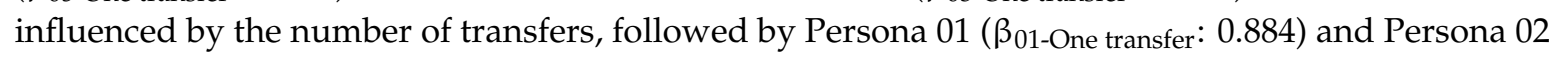

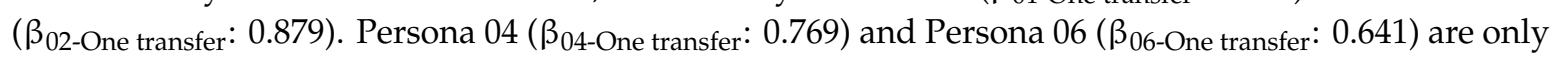
more sensitive to the number of transfers than Persona 05.

Likewise, all seven personas are positively affected by reducing the number of transfers from

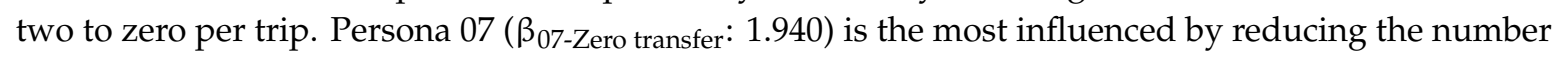

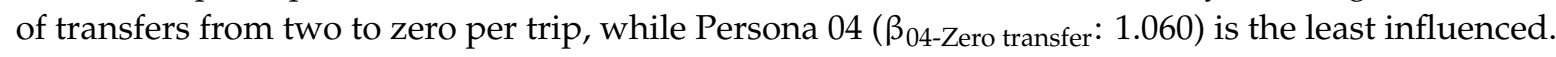

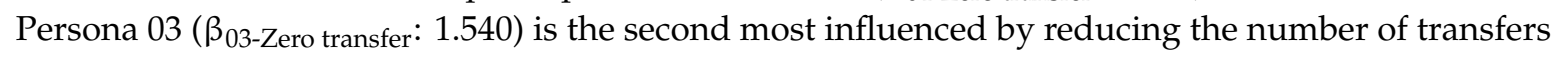

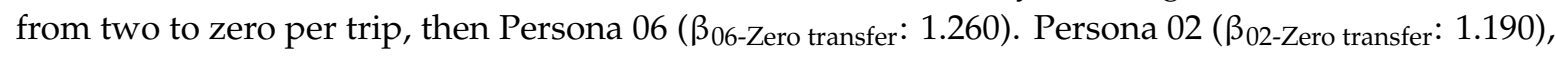

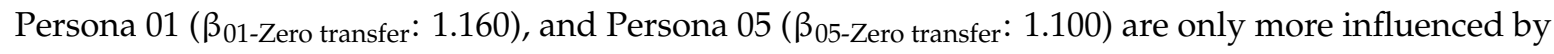
reducing the number of transfers from two to zero than Persona 04. It is worth noting that all personas prefer zero transfer trips over one transfer trip.

With regard to the provision of real-time information, transit utility for all personas is positively influenced by the provision of onboard real-time information. Persona 05 ( $\beta_{05}$-Onboard real-time: 0.504$)$ is

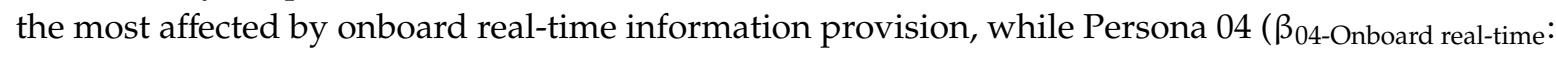

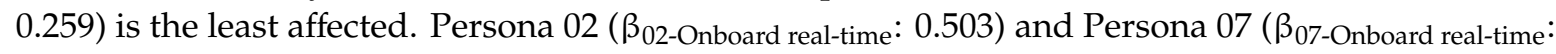
$0.467)$ are the second and third highest influenced by onboard real-time information provision,

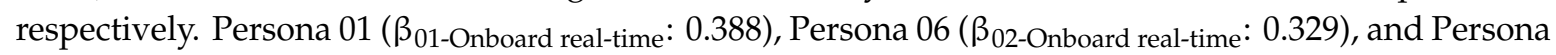
03 ( $\left.\beta_{02-\text {-Onboard real-time: }} 0.321\right)$ are only more influenced by onboard real-time information provisionthan Persona 04.

As well, all seven personas are positively affected by the provision of at-stop real-time information.

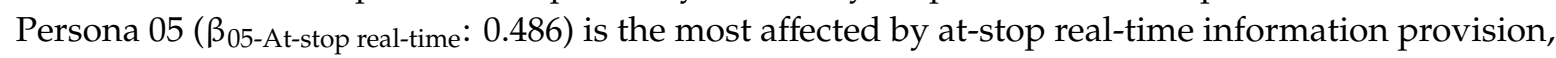

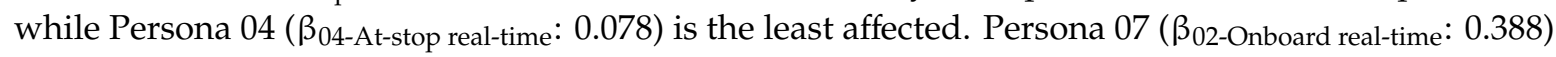

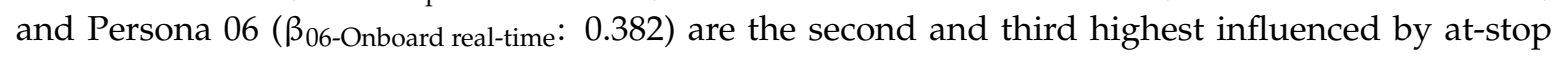

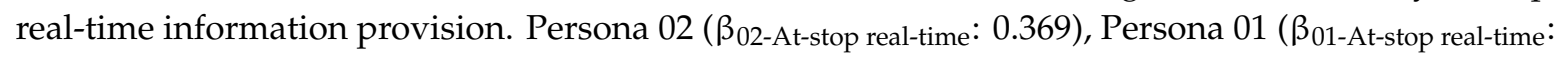

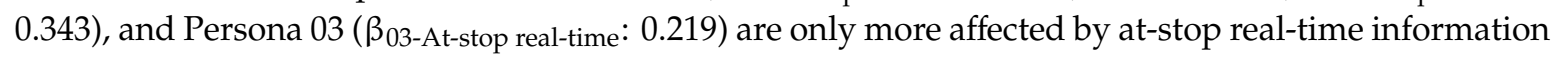
provision than Persona 04. It is worth noting that all personas prefer onboard real-time information provision over at-stop real-time information provision except for Persona 06.

Explained differently:

- Persona 01 (Full-time employee, Transit user, Positive PBC, Live in urban areas) is negatively affected by higher trip fare (the highest among all personas), longer journey time, and longer service headway, while positively affected by real-time information provision and reducing number of transfers. Nevertheless, Persona 01 is indifferent to walking time to/from bus stops.

- Persona 02 (Student, Transit user, Positive PBC, Live in urban areas) is negatively affected by longer service headway (the highest among all personas), higher trip fare, longer journey time, 
and longer walking time, while positively affected by real-time information provision (the highest among all personas regarding onboard real-time info.) and reducing number of transfers.

- Persona 03 (Full-time employee, Car driver, Neutral PBC, Live in urban areas) is negatively affected by longer journey time (the highest among all personas), higher trip fare, longer walking time (the highest among all personas), and longer service headway, while positively affected by real-time information provision and reducing number of transfers.

- Persona 04 (Retiree, Car driver, Neutral PBC, Live in urban areas) is negatively affected by longer journey time (the least among all personas), higher trip fare, longer walking time, and longer service headway (the lowest among all personas), while positively affected by real-time information provision (the lowest among all personas) and reducing number of transfers.

- Persona 05 (Student, Car Driver/Passenger, Neutral PBC, Live in urban areas) is negatively affected by longer journey time, higher trip fare, and longer service headway, while positively affected by real-time information provision (the highest among all personas regarding at-stop real-time info.) and reducing number of transfers (the lowest among all personas). However, Persona 05 is indifferent regarding walking time to/from bus stops.

- Persona 06 (Full-time employee, Car passenger, Neutral PBC, Live in urban areas) is negatively affected by longer journey time, higher trip fare, and longer service headway, while positively affected by real-time information provision and reducing number of transfers. However, walking time to/from bus stops does not prove to be of influence on this persona.

- Persona 07 (Full-time employee, Car driver, Negative PBC, Live in the suburbs) is negatively affected by longer journey time, higher trip fare (the lowest among all personas), and longer service headway, while positively affected by real-time information provision and reducing number of transfers (the highest among all personas). However, walking time to/from bus stops does not prove to be significant for Persona 07.

The visualization of the preferences of all personas for various service attributes is shown in Figure 3. It contrasts the bearing of each service attribute on the overall transit utility for all personas.

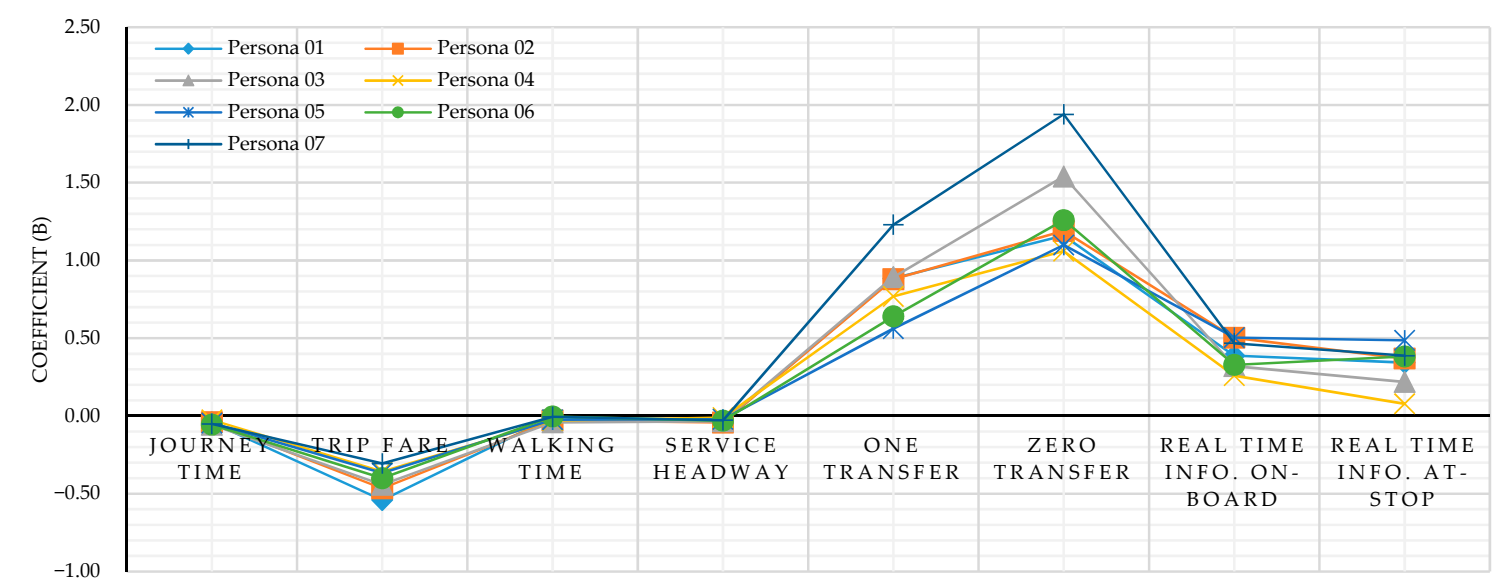

Figure 3. Bearings of service attributes on the overall transit utility across personas.

\subsection{Willingness to Pay}

The willingness to pay (WTP) for service improvements is essential for policy-makers as it enables them to adopt service improvements that are consistent with users' preferences elicited from the SP experiment. Despite the flexibility of SP experiments in testing new scenarios, proposed improvements, and a wide range of attributes/levels [60], the SP experiments have a hypothetical bias, which is grounded on the differences between the actual behaviour and the stated choices [50,61]. Loomis [61] highlighted that such a hypothetical bias might, in some cases, result in higher WTP estimates compared to real life, which should be considered by policy-makers. 
The WTP estimates were derived based on the ratio of population means, in Canadian dollars (CAD), and based on the trip fare parameter. Persona-specific WTP estimates for various service improvements are presented in Table 5 .

Table 5. WTP estimates based on the EC model.

\begin{tabular}{cccccccc}
\hline & Pers. 01 & Pers. 02 & Pers. 03 & Pers. 04 & Pers. 05 & Pers. 06 & Pers. 07 \\
\hline Reduction in Journey time (\$ per minute) & $\$ 0.076$ & $\$ 0.084$ & $\$ 0.129$ & $\$ 0.070$ & $\$ 0.125$ & $\$ 0.138$ & $\$ 0.170$ \\
Reduction in Walking time (\$ per minute) & $\$ 0.000$ & $\$ 0.062$ & $\$ 0.093$ & $\$ 0.081$ & $\$ 0.000$ & $\$ 0.000$ & $\$ 0.000$ \\
Reduction in Service headway (\$ per minute) & $\$ 0.072$ & $\$ 0.090$ & $\$ 0.077$ & $\$ 0.031$ & $\$ 0.057$ & $\$ 0.075$ & $\$ 0.092$ \\
Trip with One transfer (\$ per trip) & $\$ 1.634$ & $\$ 1.886$ & $\$ 2.023$ & $\$ 2.154$ & $\$ 1.531$ & $\$ 1.603$ & $\$ 4.020$ \\
Trip with Zero transfer (\$ per trip) & $\$ 2.144$ & $\$ 2.554$ & $\$ 3.484$ & $\$ 2.969$ & $\$ 2.997$ & $\$ 3.150$ & $\$ 6.340$ \\
Prov. of Real-time info. onboard (\$ per trip) & $\$ 0.717$ & $\$ 1.079$ & $\$ 0.726$ & $\$ 0.725$ & $\$ 1.373$ & $\$ 0.823$ & $\$ 1.526$ \\
Prov. of Real-time info. at-stop (\$ per trip) & $\$ 0.634$ & $\$ 0.792$ & $\$ 0.495$ & $\$ 0.218$ & $\$ 1.324$ & $\$ 0.955$ & $\$ 1.268$ \\
\hline
\end{tabular}

For journey time, Persona 07 (the highest among all personas) would pay around $\$ 1.70$ to save $10 \mathrm{~min}$ in trip time, while Persona 04 (the lowest) would pay only $\$ 0.70$. The second-highest willingness to pay for a $10 \mathrm{~min}$ reduction in travel time is Persona 06 (\$1.38), then Persona 03 (\$1.29). Persona 01 and Persona 02 have the second- and third-lowest WTP estimates, $\$ 0.76$ and $\$ 0.84$, respectively, for a 10 min reduction in travel time.

The WTP to save $10 \mathrm{~min}$ of walking time to/from bus stops is around $\$ 0.93$ (the highest) for Persona 03 , while around $\$ 0.62$ for Persona 02 (the lowest). Persona 04 is willing to pay $\$ 0.81$ for a 10 min reduction in walking time, while Personas $01,05,06$, and 07 are not willing to pay anything for a walking time reduction, as walking time to/from bus stops is not significantly affecting their choice.

Regarding reducing service headway (time between consecutive buses) by $10 \mathrm{~min}$, Personas 02 and 07 are willing to pay around $\$ 0.90$ (the highest), while Persona 04 would pay only $\$ 0.30$ (the lowest). Persona 03 and Persona 02, which have the second- and third-highest estimates, respectively, would pay $\$ 0.77$ and $\$ 0.75$ to reduce service headway by $10 \mathrm{~min}$. Persona 01 is willing to pay $\$ 0.72$ for reducing service headway by $10 \mathrm{~min}$. The second-lowest WTP estimate is associated with Persona 05, which would pay $\$ 0.57$ for a 10 min reduction in service headway.

The WTP for reducing the number of transfers from two to one per trip is around $\$ 4.00$ (the highest) for Persona 07, while \$1.53 (the lowest) for Persona 05. Persona 04 and Persona 03 are the second- and third-highest estimates, willing to pay $\$ 2.15$ and $\$ 2.00$, respectively, to reduce the number of transfers from two to one per trip. Persona 02 would pay around $\$ 1.88$ for reducing the number of transfers from two to one. Persona 06 and Persona 01, roughly the second-lowest estimates, are willing to pay around $\$ 1.60$ for reducing the number of transfers from two to one.

With respect to reducing the number of transfers from two to zero per trip, Persona 07 would pay around $\$ 6.30$ (the highest), and Persona 01 would pay around $\$ 2.14$ (the lowest). Persona 03, the second highest, would pay around $\$ 3.50$ to reduce the number of transfers from two to zero. Personas 04 and 05 are willing to pay around $\$ 3.00$ for reducing the number of transfers from two to zero, while it is slightly higher for Persona 06 (\$3.15). The second-lowest WTP estimate (\$2.55) for reducing the number of transfers from two to zero is for Persona 02.

The WTP for onboard real-time information provision is around $\$ 1.52$ (the highest) for Persona 07 and around $\$ 0.72$ (the lowest) for Personas 01,03 , and 04. Persona 05, the second highest, would pay nearly $\$ 1.37$ for onboard real-time information provision, while Persona 06 , the second lowest, would pay $\$ 0.82$. Persona 02 would pay around $\$ 1.08$ for onboard real-time information provision.

With regard to at-stop real-time information provision, Persona 05 would pay nearly $\$ 1.32$ (the highest), while Persona 04 would pay only around $\$ 0.22$ (the lowest). Persona 07 and Persona 06, the second and third highest, would pay $\$ 1.27$ and $\$ 0.95$, respectively, for at-stop real-time information provision, while Persona 02 would pay $\$ 0.79$. Persona 03 and Persona 01 , the second and third lowest, are willing to pay $\$ 0.49$ and $\$ 0.63$, respectively, for at-stop real-time information provision.

A related point to mention is that the WTP estimates for potential users (Personas 03, 04, 05, 06, and 07) are more likely to be higher than the WTP estimates for current users (Personas 01 and 
02). For instance, Persona 07 has the highest WTP for all service improvements except for the at-stop real-time information provision, whereas the highest WTP belongs to Persona 05, which is also a potential user. However, current users (Personas 01 and 02) have higher WTP estimates for at-stop real-time information provision than some potential users (Personas 03 and 04). Figure 4 shows the distribution of the WTP estimates for service improvements for all personas.

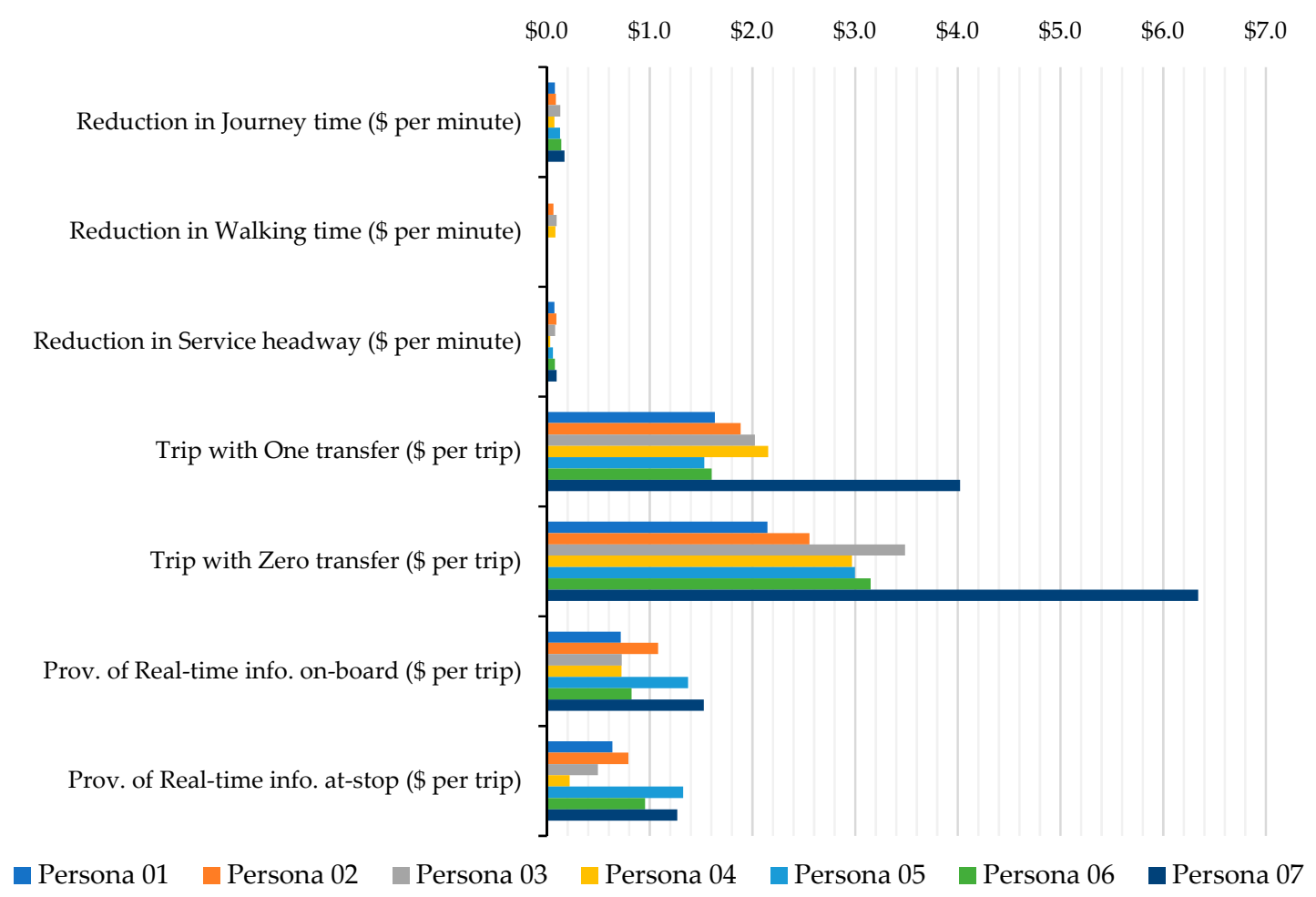

Figure 4. Distribution of WTP estimates for each Persona.

\section{Discussion of Shared and Unique Preferences}

The integration between the persona-based approach and discrete choice models presented an opportunity to further inspect the preferences of a wide spectrum of transit market segments towards service quality. Therefore, we identify the shared versus unique preferences associated with various service attributes and exhibited by each persona. Such identification enables service providers to target numerous market segments with the same service improvements.

In this respect, shared and unique preferences for service attributes are identified in the light of the statistical significance of the differences, or the lack thereof, among all personas. Statistically significant difference implies unique preferences, and insignificant difference implies shared preferences. MNL interaction models with different base categories were used, as the panel effect does not prove to be significant, to test the statistical significance of the differences among personas, as shown in Table A2-Appendix B.

Table 6, elicited from the MNL interaction models in Table A2-Appendix B, presents a mosaic of the statistically significant and insignificant differences among all seven personas, arranged with respect to each service attribute. The threshold for statistically significant differences is a $90 \%$ confidence level. 
Table 6. Unique preferences versus shared preferences (symmetric).

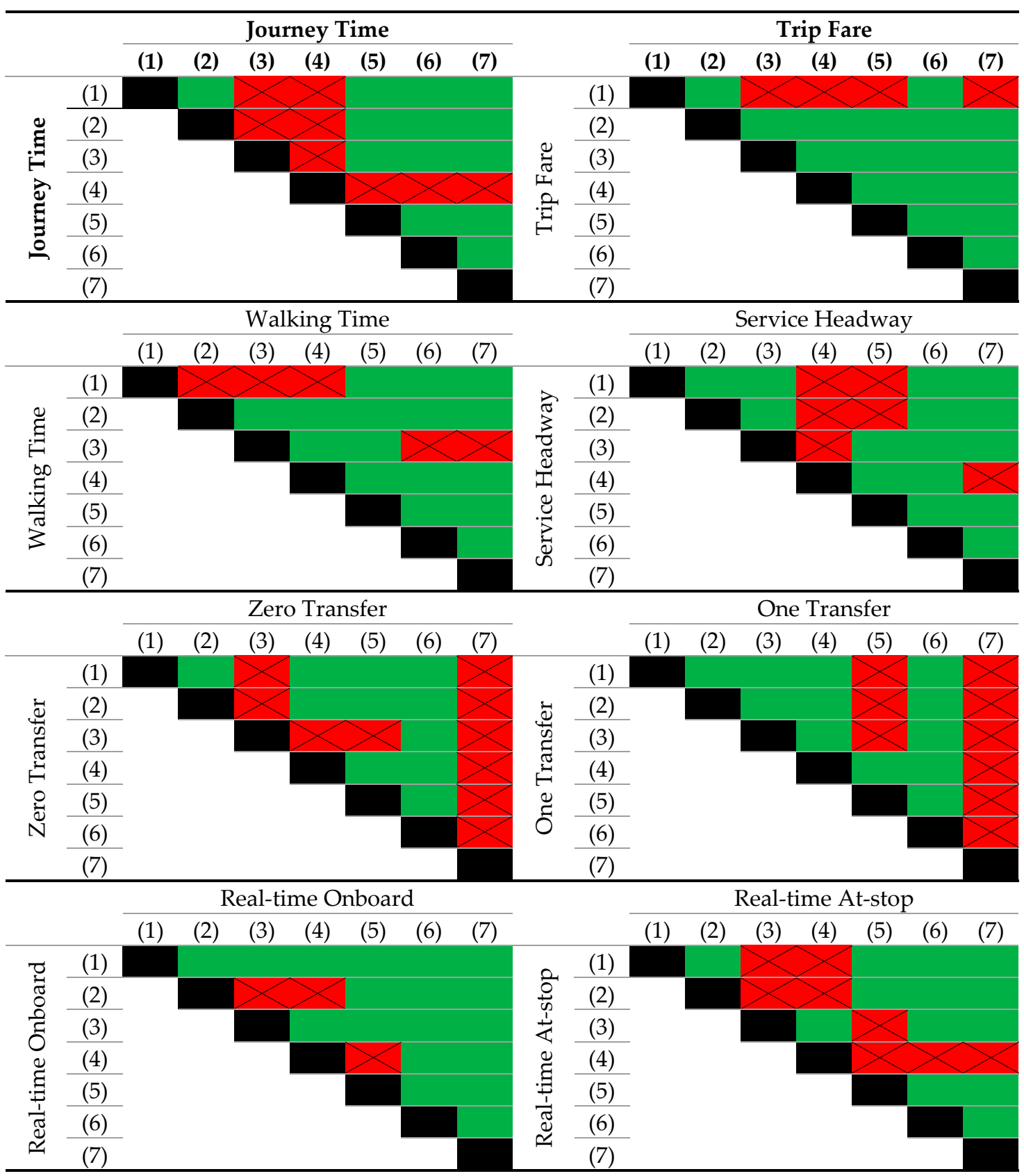

Red Crossed: Significantly different (Unique preference) and Green: Not significantly different (Mindshare).

For instance, adopting an attribute-based interpretation (e.g., journey time) of the results presented in Table 6, Personas 01, 02, 05, 06, and 07 are not significantly different from each other; therefore, they share the same preference for journey time. Whereas, Persona 04 is significantly different from all other personas, and hence it has a unique preference for transit journey time. Also, Persona 03 is significantly different from Personas 01, 02, and 04 regarding most service attributes and insignificantly different than Personas 05, 06, and 07. 
From a persona-based interpretation perspective, it could be argued that Personas 01 and 02 (both are transit users) have shared preferences regarding most service attributes, yet they have unique preference for walking time to/from bus stops. Personas 03 and 07 (both are full-time employees and car drivers) have shared preferences for many service attributes, but they have unique preferences regarding the number of transfers and walking time to/from bus stops. This might be attributed to their distinct PBC towards transit service and their different location of residence (i.e., suburbs and urban areas). Personas 02 and 05 (both are students) have shared preferences regarding all service attributes except for service headway and reducing the number of transfers from two to one per trip. Persona 07 has its own unique preference regarding the number of transfers with respect to other personas. As well, Persona 04 has a unique preference for journey time than other personas.

Overall, identifying shared preferences versus unique preferences among personas is crucial to transit agencies. Such identification enables service providers to better target their key customer segments and alter their marketing plans accordingly.

\section{Conclusions}

The study aimed at the following: first, Understanding the preferences of the dominant transit market segments considering a persona-based approach, and second, Advancing the use of the persona-based approach through quantifying personas' preferences and estimating their willingness to pay for service improvements. The study adopted an Error Component (EC) interaction model to investigate personas' preferences in a bus service desired quality choice experiment, while accounting for the panel effect, and to estimate the influence of each attribute on the overall transit utility with respect to each persona. The study adopted the preliminary seven personas, based on semistructured interviews, that best describe the key groups of the targeted transit market within the city of Hamilton. The personas capture four main characteristics: travel behaviour, employment status, geographical distribution, and Perceived Behavioural Control (PBC). The study utilized a subsample size of 2907 respondents, pulled from a larger dataset (5238), which encompasses the seven personas.

The results of the EC interaction model show that all personas are, all else being equal, negatively affected by longer journey times, higher trip fares, longer service headways, while positively affected by reducing the number of transfers per trip and real-time information provision. Nevertheless, only Personas 02,03 , and 04 are negatively affected by walking time to/from bus stops while other personas are indifferent to walking time. To use precise distinctions:

- Persona 01 (Full-time employee, Transit user, Positive PBC, Live in urban areas) is the most

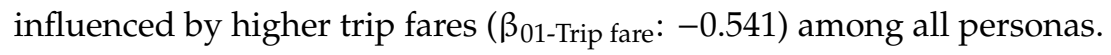

- Persona 02 (Student, Transit user, Positive PBC, Live in urban areas) is the most impacted by

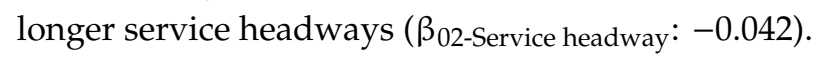

- Persona 03 (Full-time employee, Car driver, Neutral PBC, Live in urban areas) is the most affected

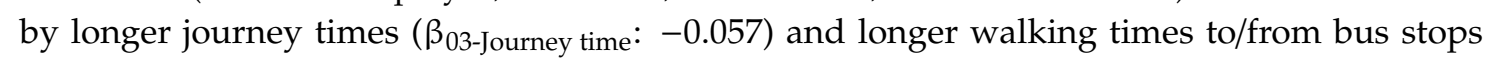

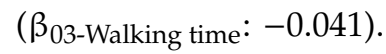

- Persona 04 (Retiree, Car driver, Neutral PBC, Live in urban areas) is the least influenced

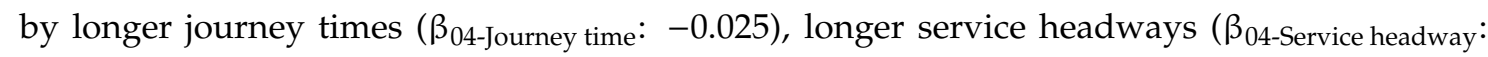

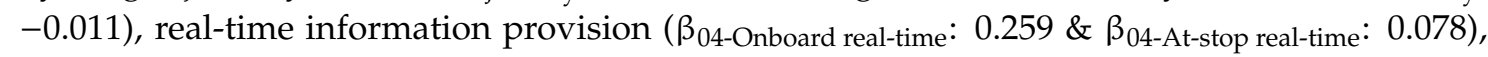

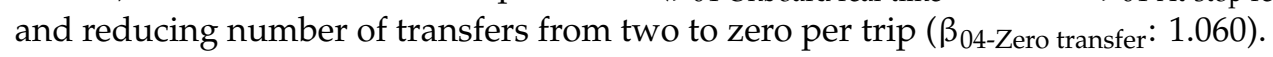

- Persona 05 (Student, Car Driver/Passenger, Neutral PBC, Live in urban areas) is the highest

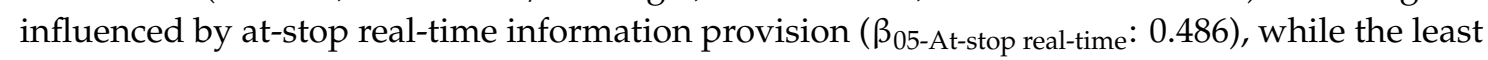
influenced by reducing number of transfers from two to one per trip ( $\beta_{05}$-One transfer: 0.562$)$.

- Persona 06 (Full-time employee, Car passenger, Neutral PBC, Live in urban areas) is among the least-affected personas regarding walking time to/from bus stops. 
- Persona 07 (Full-time employee, Car driver, Negative PBC, Live in the suburbs) is the most

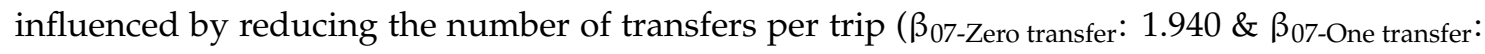
$1.230)$, and the least affected by higher trip fares $\left(\beta_{07-T r i p ~ f a r e}:-0.306\right)$.

The willingness to pay (WTP) estimates for service improvements were calculated for each persona in CAD and based on the ratio of the population means. The WTP estimates show that, in general, potential users (Personas 03, 04, 05, 06, and 07) are more likely to have higher values compared to current users (Personas 01 and 02). For instance, Persona 07 has the highest WTP for all service improvements except for the at-stop real-time information provision, where the highest WTP belongs to Persona 05, which is also a potential user. This echoes the findings of [20] that the WTP estimates for potential users are greatly higher than the estimates for current users. However, current users (Personas 01 and 02) have higher WTP estimates for at-stop real-time information provision than some potential users (Personas 03 and 04). Additionally, it is worth noting that there is no consensus within the current users (Personas 01 and 02) nor within the potential users (Personas 03, 04, 05, 06, and 07) on WTP estimates for service improvements.

Shared and unique preferences for service attributes are identified in the light of the statistical significance of the differences among personas based on MNL interaction models. The results show that Personas 01 and 02 (both are transit users) have shared preferences regarding most service attributes, yet they have unique preferences for walking time to/from bus stops. Personas 03 and 07 (both are full-time employees and car drivers) have shared preferences for many service attributes, but they have unique preferences regarding the number of transfers and walking time to/from bus stops. This might be attributed to their distinct PBC towards transit service and their different location of residence (i.e., suburbs and urban areas). Personas 02 and 05 (both are students) have shared preferences regarding various service attributes except for service headway and reducing the number of transfers from two to one per trip.

In view of the findings of this study, transit agencies should be able to tailor their improvement plans as well as their marketing/educational campaigns with a better understanding of their key customers' needs and based on quantified measures.

Author Contributions: G.E.: conceptualization, methodology, formal analysis, data curation, writing-original draft, writing-review and editing. M.M.: conceptualization, methodology, writing-original draft, writing-review and editing, supervision. All authors have read and agreed to the published version of the manuscript.

Funding: This research was funded by the City of Hamilton, Public Transit Division (Project No. Hamtn-20010000).

Acknowledgments: The authors would like to thank the reviewers for their valuable comments.

Conflicts of Interest: The authors declare no conflict of interest. 


\section{Appendix A}

Table A1. EC model estimates for personas' interactions.

\begin{tabular}{|c|c|c|c|}
\hline Variable & Coefficient $(\beta)$ & $\beta /$ Std. Err. & $p$-Value \\
\hline Journey time & -0.0412 & -9.490 & 0.000 \\
\hline Journey time $\times$ Persona 02 & 0.0025 & 0.352 & 0.725 \\
\hline Journey time $\times$ Persona 03 & -0.0153 & -2.390 & 0.017 \\
\hline Journey time $\times$ Persona 04 & 0.0167 & 2.210 & 0.027 \\
\hline Journey time $\times$ Persona 05 & -0.0045 & -0.408 & 0.683 \\
\hline Journey time $\times$ Persona 06 & -0.0139 & -0.906 & 0.365 \\
\hline Journey time $\times$ Persona 07 & -0.0108 & -0.808 & 0.419 \\
\hline Trip fare & -0.5410 & -12.500 & 0.000 \\
\hline Trip fare $\times$ Persona 02 & 0.0756 & 1.140 & 0.254 \\
\hline Trip fare $\times$ Persona 03 & 0.0990 & 1.640 & 0.102 \\
\hline Trip fare $\times$ Persona 04 & 0.1840 & 2.640 & 0.008 \\
\hline Trip fare $\times$ Persona 05 & 0.1750 & 1.720 & 0.085 \\
\hline Trip fare $\times$ Persona 06 & 0.1420 & 1.030 & 0.305 \\
\hline Trip fare $\times$ Persona 07 & 0.2350 & 2.050 & 0.041 \\
\hline Walking time & -0.0069 & -1.220 & 0.222 \\
\hline Walking time $\times$ Persona 02 & -0.0224 & -2.410 & 0.016 \\
\hline Walking time $\times$ Persona 03 & -0.0346 & -4.040 & 0.000 \\
\hline Walking time $\times$ Persona 04 & -0.0224 & -2.250 & 0.024 \\
\hline Walking time $\times$ Persona 05 & -0.0172 & -1.110 & 0.266 \\
\hline Walking time $\times$ Persona 06 & 0.0035 & 0.156 & 0.876 \\
\hline Walking time $\times$ Persona 07 & 0.0023 & 0.140 & 0.888 \\
\hline Service headway & -0.0385 & -11.200 & 0.000 \\
\hline Service headway $\times$ Persona 02 & -0.0030 & -0.539 & 0.590 \\
\hline Service headway $\times$ Persona 03 & 0.0046 & 0.921 & 0.357 \\
\hline Service headway $\times$ Persona 04 & 0.0271 & 5.060 & 0.000 \\
\hline Service headway $\times$ Persona 05 & 0.0172 & 1.980 & 0.047 \\
\hline Service headway $\times$ Persona 06 & 0.0080 & 0.790 & 0.430 \\
\hline Service headway $\times$ Persona 07 & 0.0107 & 1.270 & 0.203 \\
\hline \multicolumn{4}{|c|}{ Number of transfers (2 transfers base category) } \\
\hline One transfer & 0.8840 & 16.100 & 0.000 \\
\hline One transfer $\times$ Persona 02 & -0.0052 & -0.057 & 0.955 \\
\hline One transfer $\times$ Persona 03 & 0.0103 & 0.121 & 0.903 \\
\hline One transfer $\times$ Persona 04 & -0.1150 & -1.190 & 0.233 \\
\hline One transfer $\times$ Persona 05 & -0.3220 & -2.110 & 0.035 \\
\hline One transfer $\times$ Persona 06 & -0.2430 & -1.310 & 0.191 \\
\hline One transfer $\times$ Persona 07 & 0.3440 & 2.020 & 0.044 \\
\hline Zero transfer & 1.1600 & 14.900 & 0.000 \\
\hline Zero transfer $\times$ Persona 02 & 0.0324 & 0.256 & 0.798 \\
\hline Zero transfer $\times$ Persona 03 & 0.3840 & 3.290 & 0.001 \\
\hline Zero transfer $\times$ Persona 04 & -0.0922 & -0.708 & 0.479 \\
\hline Zero transfer $\times$ Persona 05 & -0.0610 & -0.305 & 0.760 \\
\hline Zero transfer $\times$ Persona 06 & 0.0997 & 0.386 & 0.700 \\
\hline Zero transfer $\times$ Persona 07 & 0.7820 & 3.440 & 0.001 \\
\hline \multicolumn{4}{|c|}{ Real-time information (No info. Base category) } \\
\hline Real-time info. Onboard & 0.3880 & 8.340 & 0.000 \\
\hline Real-time info. Onboard $\times$ Persona 02 & 0.1160 & 1.470 & 0.141 \\
\hline Real-time info. Onboard $\times$ Persona 03 & -0.0665 & -0.934 & 0.351 \\
\hline Real-time info. Onboard $\times$ Persona 04 & -0.1280 & -1.560 & 0.118 \\
\hline Real-time info. Onboard $\times$ Persona 05 & 0.1160 & 0.867 & 0.386 \\
\hline Real-time info. Onboard $\times$ Persona 06 & -0.0584 & -0.395 & 0.693 \\
\hline Real-time info. Onboard $\times$ Persona 07 & 0.0800 & 0.595 & 0.552 \\
\hline Real-time info. at-stop & 0.3430 & 6.500 & 0.000 \\
\hline Real-time info. at-stop $\times$ Persona 02 & 0.0262 & 0.302 & 0.762 \\
\hline Real-time info. at-stop $\times$ Persona 03 & -0.1240 & -1.530 & 0.125 \\
\hline Real-time info. at-stop $\times$ Persona 04 & -0.2650 & -2.890 & 0.004 \\
\hline Real-time info. at-stop $\times$ Persona 05 & 0.1430 & 1.020 & 0.307 \\
\hline Real-time info. at-stop $\times$ Persona 06 & 0.0395 & 0.231 & 0.818 \\
\hline Real-time info. at-stop $\times$ Persona 07 & 0.0457 & 0.308 & 0.758 \\
\hline Error Component & 0.0158 & 1.150 & 0.252 \\
\hline Log-Likelihood & & -11580.86 & \\
\hline Log-Likelihood ratio test & & 2570.716 & \\
\hline Rho-square & & 0.106 & \\
\hline
\end{tabular}

Number of respondents $=2907$, number of observations $=11,628$. 


\section{Appendix B}

Table A2. The estimation of MNL interaction models.

\begin{tabular}{|c|c|c|c|c|c|c|c|}
\hline Variable & $\begin{array}{l}\text { Persona } 01 \\
\text { (Ref.) }\end{array}$ & $\begin{array}{l}\text { Persona } \\
02 \text { (Ref.) }\end{array}$ & $\begin{array}{l}\text { Persona } \\
03 \text { (Ref.) }\end{array}$ & $\begin{array}{l}\text { Persona } \\
04 \text { (Ref.) }\end{array}$ & $\begin{array}{l}\text { Persona } \\
05 \text { (Ref.) }\end{array}$ & $\begin{array}{l}\text { Persona } \\
06 \text { (Ref.) }\end{array}$ & $\begin{array}{l}\text { Persona } 07 \\
\quad \text { (Ref.) }\end{array}$ \\
\hline Journey time $\times$ Persona 01 & $-0.041^{* * *}$ & -0.003 & $0.015^{* *}$ & $-0.017^{* *}$ & 0.005 & 0.014 & 0.011 \\
\hline Journey time $\times$ Persona 02 & 0.003 & $-0.039 * * *$ & $0.018^{* *}$ & $-0.014 *$ & 0.007 & 0.016 & 0.013 \\
\hline Journey time $\times$ Persona 03 & $-0.015^{* *}$ & $-0.018^{* *}$ & $-0.057 * * *$ & $-0.032 * * *$ & -0.011 & -0.001 & -0.004 \\
\hline Journey time $\times$ Persona 04 & $0.017 * *$ & 0.014 * & $0.032 * * *$ & $-0.025^{* * *}$ & $0.021 *$ & $0.031^{* *}$ & $0.028 * *$ \\
\hline Journey time $\times$ Persona 05 & -0.005 & -0.007 & 0.011 & $-0.021 *$ & $-0.046^{* * *}$ & 0.009 & 0.006 \\
\hline Journey time $\times$ Persona 06 & -0.014 & -0.016 & 0.001 & $-0.031 * *$ & -0.009 & $-0.055^{* * *}$ & -0.003 \\
\hline Journey time $\times$ Persona 07 & -0.011 & -0.013 & 0.004 & $-0.028 * *$ & -0.006 & 0.003 & $-0.052 * * *$ \\
\hline Trip fare $\times$ Persona 01 & $-0.541 * * *$ & -0.076 & $-0.099 *$ & $-0.184^{* * *}$ & $-0.175 *$ & -0.142 & $-0.235^{* *}$ \\
\hline Trip fare $\times$ Persona 02 & 0.076 & $-0.466^{* * *}$ & -0.023 & -0.109 & -0.099 & -0.066 & -0.159 \\
\hline Trip fare $\times$ Persona 03 & $0.099 *$ & 0.023 & $-0.442^{* * *}$ & -0.085 & -0.076 & -0.043 & -0.136 \\
\hline Trip fare $\times$ Persona 04 & $0.184 * * *$ & 0.109 & 0.085 & $-0.357 * * *$ & 0.010 & 0.043 & -0.051 \\
\hline Trip fare $\times$ Persona 05 & $0.175 *$ & 0.099 & 0.076 & -0.010 & $-0.367^{* * *}$ & 0.033 & -0.060 \\
\hline Trip fare $\times$ Persona 06 & 0.142 & 0.066 & 0.043 & -0.043 & -0.033 & $-0.400^{* * *}$ & -0.094 \\
\hline Trip fare $\times$ Persona 07 & $0.235^{* *}$ & 0.159 & 0.136 & 0.051 & 0.060 & 0.094 & $-0.306^{* * *}$ \\
\hline Walking time $\times$ Persona 01 & -0.007 & $0.022 * *$ & $0.035^{* * *}$ & $0.022 * *$ & 0.017 & -0.004 & -0.002 \\
\hline Walking time $\times$ Persona 02 & $-0.022 * *$ & $-0.029 * * *$ & 0.012 & 0.000 & -0.005 & -0.026 & -0.025 \\
\hline Walking time $\times$ Persona 03 & $-0.035^{* * *}$ & -0.012 & $-0.041^{* * *}$ & -0.012 & -0.017 & $-0.038 *$ & $-0.037^{* *}$ \\
\hline Walking time $\times$ Persona 04 & $-0.022 * *$ & 0.000 & 0.012 & $-0.029^{* * *}$ & -0.005 & -0.026 & -0.025 \\
\hline Walking time $\times$ Persona 05 & -0.017 & 0.005 & 0.017 & 0.005 & $-0.024 *$ & -0.021 & -0.020 \\
\hline Walking time $\times$ Persona 06 & 0.004 & 0.026 & $0.038 *$ & 0.026 & 0.021 & -0.003 & 0.001 \\
\hline Walking time $\times$ Persona 07 & 0.002 & 0.025 & $0.037^{* *}$ & 0.025 & 0.020 & -0.001 & -0.005 \\
\hline Service headway $\times$ Persona 01 & $-0.039 * * *$ & 0.003 & -0.005 & $-0.027^{* * *}$ & $-0.017 * *$ & -0.008 & -0.011 \\
\hline Service headway $\times$ Persona 02 & -0.003 & $-0.042^{* * * *}$ & -0.008 & $-0.030^{* * *}$ & $-0.020 * *$ & -0.011 & -0.014 \\
\hline Service headway $\times$ Persona 03 & 0.005 & 0.008 & $-0.034^{* * *}$ & $-0.022 * * *$ & -0.013 & -0.003 & -0.006 \\
\hline Service headway $\times$ Persona 04 & $0.027^{* * *}$ & $0.030 * * *$ & $0.022 * * *$ & $-0.011^{* *}$ & 0.010 & 0.019 & 0.016 * \\
\hline Service headway $\times$ Persona 05 & $0.017 * *$ & $0.020 * *$ & 0.013 & -0.010 & $-0.021^{* * *}$ & 0.009 & 0.006 \\
\hline Service headway $\times$ Persona 06 & 0.008 & 0.011 & 0.003 & -0.019 & -0.009 & $-0.030^{* * *}$ & -0.003 \\
\hline Service headway $\times$ Persona 07 & 0.011 & 0.014 & 0.006 & $-0.016 *$ & -0.006 & 0.003 & $-0.028 * * *$ \\
\hline \multicolumn{8}{|c|}{ Number of transfers (2 transfers base category) } \\
\hline One transfer $\times$ Persona 01 & $0.884^{* * *}$ & 0.005 & -0.010 & 0.115 & 0.322 ** & 0.243 & $-0.344^{* *}$ \\
\hline One transfer $\times$ Persona 02 & -0.005 & $0.879^{* * *}$ & -0.016 & 0.110 & $0.317 * *$ & 0.238 & $-0.349 * *$ \\
\hline One transfer $\times$ Persona 03 & 0.010 & 0.016 & $0.894^{* * *}$ & 0.125 & $0.332 * *$ & 0.253 & $-0.334^{* *}$ \\
\hline One transfer $\times$ Persona 04 & -0.115 & -0.110 & -0.125 & $0.769^{* * *}$ & 0.207 & 0.128 & $-0.459 * * *$ \\
\hline One transfer $\times$ Persona 05 & $-0.322 * *$ & $-0.317 * *$ & $-0.332 * *$ & -0.207 & $0.562 * * *$ & -0.079 & $-0.666^{* * *}$ \\
\hline One transfer $\times$ Persona 06 & -0.243 & -0.238 & -0.253 & -0.128 & 0.079 & $0.641^{* * *}$ & $-0.587^{* * *}$ \\
\hline One transfer $\times$ Persona 07 & $0.344^{* *}$ & $0.349 * *$ & $0.334 * *$ & $0.459^{* * *}$ & $0.666^{* * *}$ & $0.587^{* * *}$ & $1.230^{* * *}$ \\
\hline Zero transfer $\times$ Persona 01 & $1.160^{* * *}$ & -0.032 & $-0.384^{* * *}$ & 0.092 & 0.061 & -0.100 & $-0.782 * * *$ \\
\hline Zero transfer $\times$ Persona 02 & 0.032 & $1.190^{* * *}$ & $-0.352^{* * *}$ & 0.125 & 0.093 & -0.067 & $-0.750 * * *$ \\
\hline Zero transfer $\times$ Persona 03 & $0.384^{* * *}$ & $0.352 * * *$ & $1.540^{* * *}$ & $0.476^{* * *}$ & $0.445^{* *}$ & 0.284 & $-0.398 *$ \\
\hline Zero transfer $\times$ Persona 04 & -0.092 & -0.125 & $-0.476^{* * *}$ & $1.060 * * *$ & -0.031 & -0.192 & $-0.875^{* * *}$ \\
\hline Zero transfer $\times$ Persona 05 & -0.061 & -0.093 & $-0.445^{* *}$ & 0.031 & $1.100 * * *$ & -0.161 & $-0.843^{* * *}$ \\
\hline Zero transfer $\times$ Persona 06 & 0.100 & 0.067 & -0.284 & 0.192 & 0.161 & $1.260 * * *$ & $-0.683^{* *}$ \\
\hline Zero transfer $\times$ Persona 07 & $0.782 * * *$ & $0.750 * * *$ & $0.398^{*}$ & $0.875^{* * *}$ & $0.843^{* * *}$ & $0.683^{* *}$ & $1.940^{* * *}$ \\
\hline \multicolumn{8}{|c|}{ Real-time information (No info. base-category) } \\
\hline Real-time info. Onboard $\times$ Persona 01 & $0.388^{* * *}$ & -0.116 & 0.067 & 0.128 & -0.116 & 0.058 & -0.080 \\
\hline Real-time info. Onboard $\times$ Persona 02 & 0.116 & $0.503^{* * *}$ & $0.182 * *$ & $0.244^{* * *}$ & -0.001 & 0.174 & 0.036 \\
\hline Real-time info. Onboard $\times$ Persona 03 & -0.067 & $-0.182 * *$ & $0.321^{* * *}$ & 0.062 & -0.183 & -0.008 & -0.146 \\
\hline Real-time info. Onboard $\times$ Persona 04 & -0.128 & $-0.244^{* * *}$ & -0.062 & $0.259^{* * *}$ & $-0.245^{*}$ & -0.070 & -0.208 \\
\hline Real-time info. Onboard $\times$ Persona 05 & 0.116 & 0.001 & 0.183 & 0.245 * & $0.504^{* * *}$ & 0.175 & 0.036 \\
\hline Real-time info. Onboard $\times$ Persona 06 & -0.058 & -0.174 & 0.008 & 0.070 & -0.175 & $0.329 * *$ & -0.138 \\
\hline Real-time info. Onboard $\times$ Persona 07 & 0.080 & -0.036 & 0.146 & 0.208 & -0.036 & 0.138 & $0.467 * * *$ \\
\hline Real-time info. at-stop $\times$ Persona 01 & $0.343 * * *$ & -0.026 & 0.124 * & $0.265^{* * *}$ & -0.143 & -0.040 & -0.046 \\
\hline Real-time info. at-stop $\times$ Persona 02 & 0.026 & $0.369^{* * *}$ & $0.150 *$ & $0.291^{* * *}$ & -0.117 & -0.013 & -0.020 \\
\hline Real-time info. at-stop $\times$ Persona 03 & $-0.124 *$ & $-0.150 *$ & $0.219^{* * *}$ & 0.141 & $-0.267 * *$ & -0.163 & -0.170 \\
\hline Real-time info. at-stop $\times$ Persona 04 & $-0.265^{* * *}$ & $-0.291 * * *$ & -0.141 & 0.078 & $-0.408^{* * *}$ & -0.304 * & $-0.311^{* *}$ \\
\hline Real-time info. at-stop $\times$ Persona 05 & 0.143 & 0.117 & $0.267^{* *}$ & $0.408^{* * *}$ & $0.486^{* * *}$ & 0.104 & 0.098 \\
\hline Real-time info. at-stop $\times$ Persona 06 & 0.040 & 0.013 & 0.163 & $0.304 *$ & -0.104 & $0.382 * *$ & -0.006 \\
\hline Real-time info. at-stop $\times$ Persona 07 & 0.046 & 0.020 & 0.170 & $0.311 * *$ & -0.098 & 0.006 & $0.388^{* * *}$ \\
\hline Log-likelihood & & & & $-11,580.88$ & & & \\
\hline Log-likelihood ratio test & & & & 2387.56 & & & \\
\hline Rho-square & & & & 0.0934 & & & \\
\hline
\end{tabular}

Note: ${ }^{* * *}, * *$, and ${ }^{*}$ indicate significance at $1 \%, 5 \%$, and $10 \%$ levels, respectively. 


\section{References}

1. Eboli, L.; Mazzulla, G. Discrete choice models as a tool for transit service quality evaluation. Electron. J. Appl. Stat. Anal. Decis. Support Syst. Serv. Eval. 2011, 2, 65-73. [CrossRef]

2. De Oña, J.; De Oña, R.; Calvo, F.J. A classification tree approach to identify key factors of transit service quality. Expert Syst. Appl. 2012, 39, 11164-11171. [CrossRef]

3. Allen, J.; Muñoz, J.C.; de Dios Ortúzar, J. Modelling service-specific and global transit satisfaction under travel and user heterogeneity. Transp. Res. Part A Policy Pract. 2018, 113, 509-528. [CrossRef]

4. Mahmoud, M.; Hine, J. Using AHP to measure the perception gap between current and potential users of bus services. Transp. Plan. Technol. 2013, 36, 4-23. [CrossRef]

5. Mahmoud, M.; Hine, J. Measuring the influence of bus service quality on the perception of users. Transp. Plan. Technol. 2016, 39, 284-299. [CrossRef]

6. Abenoza, R.F.; Cats, O.; Susilo, Y.O. Travel satisfaction with public transport: Determinants, user classes, regional disparities and their evolution. Transp. Res. Part A Policy Pract. 2017, 95, 64-84. [CrossRef]

7. Deb, S.; Ali Ahmed, M. Determining the service quality of the city bus service based on users' perceptions and expectations. Travel Behav. Soc. 2018, 12,1-10. [CrossRef]

8. Beimborn, E.A.; Greenwald, M.J.; Jin, X. Accessibility, Connectivity, and Captivity Impacts on Transit Choice. Transp. Res. Rec. 2003, 1835, 1-9. [CrossRef]

9. Krizek, K.; El-Geneidy, A. Segmenting preferences and habits of transit users and non-users. J. Public Transp. 2007, 10, 71-94. [CrossRef]

10. Venter, C. Are We Giving Brt Passengers What They Want? User Preference and Market Segmentation in Johannesburg. In Proceedings of the 35th Southern African Transport Conference (SATC 2016), Pretoria, South Africa, 4-7 July 2016; pp. 658-672.

11. Van Lierop, D.; El-Geneidy, A. A new market segmentation approach: Evidence from two Canadian cities. J. Public Transp. 2017, 20, 20-43. [CrossRef]

12. Grisé, E.; El-geneidy, A. Where is the happy transit rider? Evaluating satisfaction with regional rail service using a spatial segmentation approach. Transp. Res. Part A 2018, 114, 84-96. [CrossRef]

13. Eboli, L.; Forciniti, C.; Mazzulla, G. Spatial variation of the perceived transit service quality at rail stations. Transp. Res. Part A Policy Pract. 2018, 114, 67-83. [CrossRef]

14. Nikel, C.; Eldeeb, G.; Mohamed, M. Perceived Quality of Bus Transit Services: A Route-Level Analysis. Transp. Res. Rec. J. Transp. Res. Board 2020, 2674. [CrossRef]

15. De Oña, R.; López, G.; de los Rios, F.J.D.; de Oña, J. Cluster Analysis for Diminishing Heterogeneous Opinions of Service Quality Public Transport Passengers. Procedia Soc. Behav. Sci. 2014, 162, 459-466. [CrossRef]

16. De Oña, J.; de Oña, R.; López, G. Transit service quality analysis using cluster analysis and decision trees: A step forward to personalized marketing in public transportation. Transportation 2016, 43, 725-747. [CrossRef]

17. Dell'Olio, L.; Ibeas, A.; Cecin, P. The quality of service desired by public transport users. Transp. Policy 2011, 18, 217-227. [CrossRef]

18. Eboli, L.; Forciniti, C.; Mazzulla, G.; Calvo, F. Exploring the Factors That Impact on Transit Use through an Ordered Probit Model: The Case of Metro of Madrid. Transp. Res. Procedia 2016, 18, 35-43. [CrossRef]

19. Eboli, L.; Mazzulla, G. Willingness-to-pay of public transport users for improvement in service quality. Eur. Transp. 2008, 38, 107-118.

20. Bellizzi, M.G.; Dell'Olio, L.; Eboli, L.; Mazzulla, G. Heterogeneity in desired bus service quality from users' and potential users' perspective. Transp. Res. Part A 2020, 132, 365-377. [CrossRef]

21. Eldeeb, G.; Mohamed, M. Quantifying Preference Heterogeneity in Transit Service Desired Quality Using Random Parameter Logit and Latent Class Choice Models. Transp. Res. Part A J. 2020. under review.

22. Metrolinx. 2041 Regional Transportation Plan; Greater Toronto and Hamilton Area, ON, Canada, 2018; ISBN 9781775137924.

23. OXD TransLink Customer Experience Research. Available online: https://oxd.com/work/translink-customerexperience-research/ (accessed on 7 March 2020).

24. UX Planet Using Design Thinking to Improve Bus Accessibility. Available online: https://uxplanet.org/uxfor-a-better-public-transport-23b81ccc56de (accessed on 7 March 2020). 
25. Fergnani, A. The future persona: A futures method to let your scenarios come to life. Foresight 2019, 21, 445-466. [CrossRef]

26. Cooper, A. The Inmates Are Running The Asylum; Sams: Indianapolis, IN, USA, 1999; Volume 43, ISBN 0-672-32614-0.

27. Miaskiewicz, T.; Kozar, K.A. Personas and user-centered design: How can personas benefit product design processes? Des. Stud. 2011, 32, 417-430. [CrossRef]

28. Nielsen, L. Personas—User Focused Design, 1st ed.; Springer: London, UK, 2013; ISBN 978-1-4471-5903-2.

29. Pruitt, J.; Grudin, J. Personas: Practice and Theory. In Proceedings of the 2003 Conference on Designing for User Experiences, San Francisco, CA, USA, June 2003; pp. 1-15.

30. Miaskiewicz, T.; Luxmoore, C. The Use of Data-Driven Personas to Facilitate Organizational Adoption-A Case Study. Des. J. 2017, 20, 357-374. [CrossRef]

31. Marshall, R.; Cook, S.; Mitchell, V.; Summerskill, S.; Haines, V.; Maguire, M.; Sims, R.; Gyi, D.; Case, K. Design and evaluation: End users, user datasets and personas. Appl. Ergon. 2015, 46, 311-317. [CrossRef]

32. Beyer, S.; Müller, A. Evaluation of Persona-Based User Scenarios in Vehicle Development. In Proceedings of the Human Systems Engineering and Design; Ahram, T., Karwowski, W., Taiar, R., Eds.; Springer International Publishing: Cham, Switzerland, 2018; pp. 750-756.

33. Vosbergen, S.; Mulder-Wiggers, J.M.R.; Lacroix, J.P.; Kemps, H.M.C.; Kraaijenhagen, R.A.; Jaspers, M.W.M.; Peek, N. Using personas to tailor educational messages to the preferences of coronary heart disease patients. J. Biomed. Inform. 2015, 53, 100-112. [CrossRef]

34. Boyce, R.D.; Ragueneau-Majlessi, I.; Yu, J.; Tay-Sontheimer, J.; Kinsella, C.; Chou, E.; Brochhausen, M.; Judkins, J.; Gufford, B.T.; Pinkleton, B.E.; et al. Developing User Personas to Aid in the Design of a User-Centered Natural Product-Drug Interaction Information Resource for Researchers. AMIA Annu. Symp. Proc. 2018, 2018, 279-287.

35. Jones, M.C.; Floyd, I.R.; Twidale, M.B. Teaching Design with Personas. Interact. Des. Archit. 2008, 3-4, 75-82.

36. Canham, S.L.; Mahmood, A. The use of personas in gerontological education. Gerontol. Geriatr. Educ. 2019, 40, 468-479. [CrossRef]

37. Lindgren, A.; Chen, F.; Amdahl, P.; Chaikiat, P. Using Personas and Scenarios as an Interface Design Tool for Advanced Driver Assistance Systems. In Proceedings of the Universal Access in Human-Computer Interaction. Ambient Interaction; Stephanidis, C., Ed.; Springer: Berlin/Heidelberg, Germany, 2007; pp. 460-469.

38. Schäfer, C.; Zinke, R.; Künzer, L.; Hofinger, G.; Koch, R. Applying persona method for describing users of escape routes. Transp. Res. Procedia 2014, 2, 636-641. [CrossRef]

39. De Clerck, Q.; van Lier, T.; Messagie, M.; Macharis, C.; Van Mierlo, J.; Vanhaverbeke, L. Total Cost for Society: A persona-based analysis of electric and conventional vehicles. Transp. Res. Part D Transp. Environ. 2018, 64, 90-110. [CrossRef]

40. Kong, P.; Cornet, H.; Frenkler, F. Personas and Emotional Design for Public Service Robots: A Case Study with Autonomous Vehicles in Public Transportation. In Proceedings of the 2018 International Conference on Cyberworlds (CW), Singapore, 3-5 October 2018; pp. 284-287. [CrossRef]

41. Chapman, C.N.; Milham, R.P. The personas' new clothes: Methodological and practical arguments against a popular method. Proc. Hum. Factors Ergon. Soc. 2006, 50, 634-636. [CrossRef]

42. Turner, P.; Turner, S. Is stereotyping inevitable when designing with personas? Des. Stud. 2011, 32, 30-44. [CrossRef]

43. Madsen, A.; Mckagan, S.B.; Sayre, E.C.; Martinuk, M.; Bell, A. Personas as a Powerful Methodology to Design Targeted Professional Development Resources Methodology: Creation of Personas. arXiv 2014, arXiv:1408.1125v2.

44. Chapman, C.N.; Love, E.; Milham, R.P.; Elrif, P.; Alford, J.L. Quantitative evaluation of personas as information. Proc. Hum. Factors Ergon. Soc. 2008, 2, 1107-1111. [CrossRef]

45. Ben-Akiva, M.; Bolduc, D. Multinomial Probit with a Logit Kernel and a General Parametric Specification of the Covariance Structure; 1996. Available online: https://eml.berkeley.edu/reprints/misc/multinomial.pdf. (accessed on 13 April 2020).

46. McFadden, D.; Train, K. Mixed MNL models for discrete response. J. Appl. Econom. 2000, 15, 447-470. [CrossRef]

47. Ben-Akiva, M.; Lerman, S. Discrete Choice Analysis Theory and Application to Travel Deamnd, 3rd ed.; The Massachusetts Institute of Technology: Cambridge, MA, USA; London, UK, 1985; ISBN 0-262-02217-6. 
48. McFadden, D. Measuring willingness to pay for transportation improvements. Theor. Found. Travel Choice Model. 1998, 339, 364.

49. Rizzi, L.I.; Ortúzar, J.D.D. Stated preference in the valuation of interurban road safety. Accid. Anal. Prev. 2003, 35, 9-22. [CrossRef]

50. Ortúzar, J.D.D.; Willumsen, L.G. Modelling Transport, 4th ed.; John Wiley \& Sons, Ltd.: West Sussex, UK, 2011; ISBN 9780470760390.

51. Hensher, D.A.; Greene, W.H. The Mixed Logit model: The state of practice. Transportation 2003, 30, $133-176$. [CrossRef]

52. Bierlaire, M. PandasBiogeme: A Short Introduction; Technical report TRANSP-OR 181219. Transport and Mobility Laboratory, ENAC, EPFL; Switzerland, 2018.

53. Hess, S.; Train, K.E.; Polak, J.W. On the use of a Modified Latin Hypercube Sampling (MLHS) method in the estimation of a Mixed Logit Model for vehicle choice. Transp. Res. Part B Methodol. 2006, 40, 147-163. [CrossRef]

54. Hensher, D.A.; Rose, J.M.; Greene, W.H. Applied Choice Analysis A Primer, 1st ed.; Cambridge University Press: Cambridge, UK, 2005; ISBN 13 978-0-521-84426-0.

55. City of Hamilton Hamilton Street Railway. Available online: https://www.hamilton.ca/hsr-bus-schedulesfares (accessed on 6 June 2018).

56. Eldeeb, G.; Nikel, C.; Ferguson, M.; Mohamed, M. Service Quality and Consumers Preferences for Hamilton Street Railway (HSR); City of Hamilton, ON, Canada, 2019. Available online: https://www.researchgate.net/publication/338750510_Service_Quality_and_Consumers_Preferences_ for_Hamilton_Street_Railway_HSR_-_Executive_Summary (accessed on 13 April 2020).

57. Bliemer, M.C.J.; Rose, J.M. Designing Stated Choice Experiments: State-of-the-Art; Emerald: Bingley, UK, 2006.

58. Kuhfeld, W.; Tobias, R.; Garratt, M. Efficient experimental design with marketing research applications. J. Mark. Res. 1994, 31, 545-557. [CrossRef]

59. Mohamed, M.; Higgins, C.; Ferguson, M.; Kanaroglou, P. Identifying and characterizing potential electric vehicle adopters in Canada: A two-stage modelling approach. Transp. Policy 2016, 52, 100-112. [CrossRef]

60. Hensher, D. Stated preference analysis of travel choice: The state of practice. Transportation 1994, 21, 107-133. [CrossRef]

61. Loomis, J.B. Strategies for overcoming hypothetical bias in stated preference surveys. J. Agric. Resour. Econ. 2014, 39, 34-46. [CrossRef] 